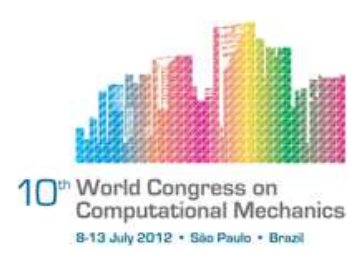

\title{
THE NUMERICAL PREDICTION OF RAILWAY INDUCED VIBRATIONS IN AN URBAN ENVIRONMENT
}

\author{
P. Coulier, S. François, G. Lombaert, G. Degrande \\ Department of Civil Engineering, KU Leuven \\ Kasteelpark Arenberg 40, B-3001 Heverlee \\ pieter.coulier@bwk.kuleuven.be
}

\begin{abstract}
Boundary element methods are often employed in the numerical prediction of railway induced vibrations. The evaluation of large models is limited, however, due to the computational requirements in terms of memory storage and computation time, which impedes to rigorously account for the presence of multiple buildings in an urban area. This paper presents a novel hierarchical boundary element method for elastodynamics based on halfspace Green's functions; meshing of the free surface and the layer interfaces is hence avoided. Numerical examples are discussed to demonstrate the effectiveness of the methodology, indicating that the model size can be increased with an order of magnitude, which allows to perform large scale boundary element computations. A synthetic case study of an urban environment is finally introduced to investigate the influence of the trough-soil coupling of closely spaced buildings on the wavefield in the soil and the structural response.
\end{abstract}

Keywords: Boundary element method, $\mathscr{H}$-matrices, elastodynamics, halfspace Green's functions, railway induced vibrations.

\section{INTRODUCTION}

The numerical prediction of vibrations in buildings due to railway traffic is a problem of dynamic soil-structure interaction (SSI) where wave propagation in the soil couples the source (track/tunnel) and the receivers (surrounding buildings). In the past decades, several numerical models have been developed $[1,20]$. Analytical or finite element (FE) models are used for the track/tunnel and coupled to a boundary element (BE) model for the soil to account for dynamic SSI. In order to avoid the meshing of the free surface and the soil layer interfaces, the use of Green's functions for a horizontally layered halfspace in the BE formulation is preferred. Innovative numerical techniques have been developed to avoid a full three-dimensional (3D) model of the structure. For structures exhibiting a regular geometry such as railways, a significant reduction in computation time can be obtained. If longitudinally invariant structures are dealt with, a Fourier transform of the longitudinal coordinate is appropriate, while the Floquet transform can be applied if periodic structures are considered. 
Although the aforementioned innovative numerical techniques enhance the applicability of the numerical models, the evaluation of large models still remains a challenge from a computational point of view, due to CPU and memory limitations. This is largely attributed to the fact that dense, fully populated unsymmetric matrices arise from the BE formulation. As a result, the state-of-the-art numerical models only allow to take the through-soil coupling of very few structures into account, and are unable to rigorously model dense urban areas characterized by many closely spaced structures.

The aim of this paper is therefore to develop a numerical model which allows to predict railway induced vibrations in an urban environment. Such a model should be based on a fast BE method in order to reduce the overall complexity. Several fast BE methods have been developed, including the fast multipole method (FMM) [23], the panel clustering technique [17] and methods based on hierarchical matrices [16]. In the FMM, the Green's functions are reformulated by using a multipole expansion, and this has proven to be very efficient in case analytical expressions of the Green's functions are at hand. The application of the FMM method to elastodynamics is therefore limited to formulations based on closed form full space fundamental solutions $[9,15]$. For problems involving a layered halfspace, however, a huge amount of boundary elements should be sacrificed in order to discretize the free surface and the layer interfaces if a BE formulation based on full space fundamental solutions is employed. The FMM method is therefore not suited in the context of railway induced vibrations. Fast $\mathrm{BE}$ methods based on hierarchical matrices in combination with efficient algorithms such as adaptive cross approximation provide an alternative to tackle problems in which analytical expressions of the Green's functions are not available, as these methods are in essence algebraic tools to approximate the BE matrices. This approach has already been applied successfully to isotropic [8] and anisotropic [21] elastodynamic problems.

To the knowledge of the authors, however, no efforts have been made in the literature to develop and apply a fast BE method for elastodynamics based on Green's functions for a horizontally layered halfspace. In this paper, such a method based on hierarchical matrices is presented and applied for the first time, which eventually allows to predict railway induced vibrations in an urban environment. The text is organized as follows. Section 2 summarizes the basic principles of hierarchical BE methods. The numerical implementation of the methodology is validated in section 3. The applicability of the novel approach to the prediction of railway induced vibrations in an urban environment is finally illustrated by means of a synthetic case study in section 4 .

\section{A HIERARCHICAL BOUNDARY ELEMENT METHOD FOR ELASTODYNAMICS BASED ON GREEN'S FUNCTIONS FOR A HORIZONTALLY LAYERED HALF- SPACE}

In the boundary element method, the boundary integral equation relating the field variables to the elastodynamic state on the boundary of the domain is discretized, resulting in a boundary element system of equations. For unbounded domains, the displacements $\underline{\hat{\mathbf{u}}}(\omega)$ and tractions $\underline{\hat{\mathbf{t}}}(\omega)$ at the collocation points are related as follows in the frequency domain:

$$
(\hat{\mathbf{T}}(\omega)+\mathbf{I}) \underline{\hat{\mathbf{u}}}(\omega)=\hat{\mathbf{U}}(\omega) \underline{\hat{\mathbf{t}}}(\omega)
$$


The system matrices $\hat{\mathbf{U}}(\omega)$ and $\hat{\mathbf{T}}(\omega)$ are in general fully populated unsymmetric matrices, while I represents a unity matrix, corresponding to the integral free term in the boundary integral equation. The latter vanishes for a bounded domain. The computation of the system matrices $\hat{\mathbf{U}}(\omega)$ and $\hat{\mathbf{T}}(\omega)$ requires integration over the Green's functions. The Green's displacements $\hat{u}_{i j}^{\mathrm{G}}\left(\mathbf{x}^{\prime}, \mathbf{x}, \omega\right)$ and tractions $\hat{t}_{i j}^{\mathrm{Gn}}\left(\mathbf{x}_{\mathrm{s}}^{\prime}, \mathbf{x}, \omega\right)$ correspond to the fundamental solutions at a receiver location $\mathbf{x}$ in a direction $\mathbf{e}_{j}$ due to a unit time-harmonic point load at a source location $\mathrm{x}^{\prime}$ in a direction $\mathbf{e}_{i}$. A quadratic amount of memory $\left(\mathcal{O}\left(N_{\text {DOF }}^{2}\right)\right)$ is required to store the system matrices $\hat{\mathbf{U}}(\omega)$ and $\hat{\mathbf{T}}(\omega)$. Furthermore, solving the set of equations (1) by means of direct numerical solvers such as $L U$-decomposition requires a cubic amount of numerical operations $\left(\mathcal{O}\left(N_{\text {DOF }}^{3}\right)\right)$. The application of the classical boundary element method is therefore currently limited to problems of an order of magnitude of $\mathcal{O}\left(10^{4}\right)$ [22].

The use of hierarchical matrices ( $\mathscr{H}$-matrices) provides an elegant way to treat fully populated matrices with almost linear complexity [13]. A $\mathscr{H}$-matrix is a data-sparse representation of a certain matrix, consisting of a collection of block matrices of various sizes. The construction of a $\mathscr{H}$-matrix requires several steps. First, a hierarchical cluster tree is constructed based on the boundary element mesh. At the lowest level (i.e. level 0), the cluster consists of the complete BE mesh. Each cluster is recursively partitioned into two (more or less equal) sons. Several techniques are available in order to obtain a suitable cluster tree (e.g. nested dissection $[12,14]$, cardinality balanced clustering [6], ... ). In what follows, a clustering strategy based on principal component analysis (PCA) will be employed [22]. In PCA, the eigenvectors of the covariance matrix of a cluster are first calculated. The eigenvector corresponding to the largest eigenvalue gives the direction of the longest expanse of the considered cluster. A separation plane orthogonal to the aforementioned eigenvector is drawn through the center of the cluster, dividing it in two (more or less equal) sons. This procedure can recursively be applied to every son, until the clusters contain less or equal elements (or nodes) than a prescribed number $N_{\text {min }}$. Second, admissible cluster pairs $(X, Y)$ are identified, i.e. cluster subdomains which satisfy a geometric admissibility criterion such that the corresponding fundamental solutions are smooth [4, 22]:

$$
\min \{\operatorname{diam}(\mathrm{X}), \operatorname{diam}(\mathrm{Y})\}<\eta \operatorname{dist}(\mathrm{X}, \mathrm{Y}) \quad 0<\eta<1
$$

where $\operatorname{diam}(\mathrm{X})$ denotes the maximal expanse of cluster $X$ and $\operatorname{dist}(\mathrm{X}, \mathrm{Y})$ is the minimal distance between clusters $X$ and $Y$. The parameter $\eta$ affects the number of admissible blocks and the quality of the approximation of the admissible pairs [21].

For an admissible cluster pair $(X, Y)$, the fundamental solutions are smooth enough such that the corresponding block BE matrices $\hat{\mathbf{T}}_{(X, Y)}(\omega) \in \mathbb{C}^{m \times n}$ and $\hat{\mathbf{U}}_{(X, Y)}(\omega) \in \mathbb{C}^{m \times n}$ can be approximated by low rank approximations. $m$ and $n$ denote the number of degrees of freedom in clusters $X$ and $Y$, respectively. For example, the block matrix $\hat{\mathbf{T}}_{(X, Y)}(\omega)$ is approximated by $\tilde{\mathbf{T}}_{(X, Y)}(\omega)$ :

$$
\hat{\mathbf{T}}_{(X, Y)}(\omega) \simeq \tilde{\mathbf{T}}_{(X, Y)}(\omega)=\left(\mathbf{U}_{\hat{\mathbf{T}}_{(X, Y)}(\omega)}\right)\left(\mathbf{V}_{\hat{\mathbf{T}}_{(X, Y)}(\omega)}\right)^{\star}
$$

with $\mathbf{U}_{\hat{\mathbf{T}}_{(X, Y)}(\omega)} \in \mathbb{C}^{m \times k}$ and $\mathbf{V}_{\hat{\mathbf{T}}_{(X, Y)}(\omega)} \in \mathbb{C}^{n \times k}$, and where $\star$ indicates the complex conjugate. $k$ is the rank of the representation. For $k(m+n)<m n, \tilde{\mathbf{T}}_{(X, Y)}(\omega)$ is called a low rank approximation of $\hat{\mathbf{T}}_{(X, Y)}(\omega)$, as the memory storage can be reduced from $\mathcal{O}(m n)$ to $\mathcal{O}(k(m+n))$ by storing $\mathbf{U}_{\hat{\mathbf{T}}_{(X, Y)}(\omega)}$ and $\mathbf{V}_{\hat{\mathbf{T}}_{(X, Y)}(\omega)}$ instead of $\hat{\mathbf{T}}_{(X, Y)}(\omega)$, which is linear in 
$m$ and $n$. The rank $k$ is determined such that the approximation $\tilde{\mathbf{T}}_{(X, Y)}(\omega)$ is accurate up to a prescribed relative accuracy $\varepsilon$ :

$$
\left\|\hat{\mathbf{T}}_{(X, Y)}(\omega)-\tilde{\mathbf{T}}_{(X, Y)}(\omega)\right\|_{\mathrm{F}} \leq \varepsilon\left\|\hat{\mathbf{T}}_{(X, Y)}(\omega)\right\|_{\mathrm{F}}
$$

where $\|\diamond\|_{F}$ indicates the Frobenius norm of the matrix $\diamond$. The partially pivoted adaptive cross approximation (ACA) algorithm [7,22] is employed to compute the low rank approximation defined in equation (3). The algorithm adaptively calculates some of the rows and columns of the original block matrix to obtain an approximation from few of the original matrix entries. The algorithm stops if the prescribed accuracy $\varepsilon$ is attained; the stopping criterion defined in equation (4) can however not be employed, as the original matrix $\hat{\mathbf{T}}_{(X, Y)}(\omega)$ is never generated completely. An intrinsic stopping criterion based on the variation of the Frobenius norm in consecutive approximations is therefore used. The amount of numerical operations required in the ACA algorithm is $\mathcal{O}\left(k^{2}(m+n)\right)$ [22]. One of the major advantages of applying the ACA algorithm to obtain low rank approximations of the BE block matrices corresponding to admissible cluster pairs is that it is a pure algebraic approach, implying that there is no need for (semi-)analytical expressions of the fundamental solutions. In this paper, Green's functions $\hat{u}_{i j}^{\mathrm{G}}\left(\mathbf{x}^{\prime}, \mathbf{x}, \omega\right)$ and $\hat{t}_{i j}^{\mathrm{Gn}} \mathbf{n}_{\mathrm{s}}\left(\mathbf{x}^{\prime}, \mathbf{x}, \omega\right)$ for a horizontally layered halfspace are employed. These Green's functions are numerically computed with the direct stiffness method [19] using the MATLAB toolbox EDT 2.2 [25]. For cluster pairs $(X, Y)$ not satisfying the admissibility criterion (2), the fundamental solutions show a singular behaviour which does not allow to construct a low rank approximation of the corresponding block BE matrices. They are therefore computed exactly.

The BE matrices $\hat{\mathbf{T}}(\omega)$ and $\hat{\mathbf{U}}(\omega)$ can hence be approximated by their hierarchical representations $\hat{\mathbf{T}}_{\mathscr{H}}(\omega)$ and $\hat{\mathbf{U}}_{\mathscr{H}}(\omega)$, respectively, and the BE set of equations (1) can finally be replaced by:

$$
\left(\hat{\mathbf{T}}_{\mathscr{H}}(\omega)+\mathbf{I}\right) \underline{\hat{\mathbf{u}}}(\omega)=\hat{\mathbf{U}}_{\mathscr{H}}(\omega) \hat{\mathbf{t}}(\omega)
$$

In order to solve the set of equations (5), iterative Krylov subspace methods are adequate, as the complexity of matrix-vector multiplications is only $\mathcal{O}\left(N_{\text {DOF }} \log N_{\text {DOF }}\right)$ for $\mathscr{H}$-matrices [6], and this operation forms the core of iterative solvers. In this paper, all sets of equations are solved by means of the generalized minimal residual method (GMRES).

It follows from the discussion above that the use of $\mathscr{H}$-matrices results in a significant reduction of memory and $\mathrm{CPU}$ requirements and hence allows to perform large scale $\mathrm{BE}$ computations.

\section{VALIDATION}

The hierarchical BE method outlined in section 2 has been implemented in the MATLAB toolbox BEMFUN [11]. In the following subsections, two examples are considered to validate the numerical implementation and to demonstrate the effectiveness of the methodology. The first example is merely included to validate the correct implementation of $\mathscr{H}-$ matrix arithmetics and does not involve Green's functions for a layered halfspace, while the second example focuses on the application of the novel hierarchical BE method, incorporating the aforementioned Green's functions.

\subsection{Three-dimensional spherical cavity subjected to an internal pressure}

A three-dimensional spherical cavity with radius $r_{0}=1 \mathrm{~m}$ in a full space loaded by an internal pressure $p=1 \mathrm{~Pa} / \mathrm{Hz}$ is investigated in this subsection. The full space is 
characterized by a shear wave velocity $C_{\mathrm{s}}=150 \mathrm{~m} / \mathrm{s}$, a dilatational wave velocity $C_{\mathrm{p}}=$ $300 \mathrm{~m} / \mathrm{s}$ and a density $\rho=1800 \mathrm{~kg} / \mathrm{m}^{3}$. No material damping is taken into account.

(a)
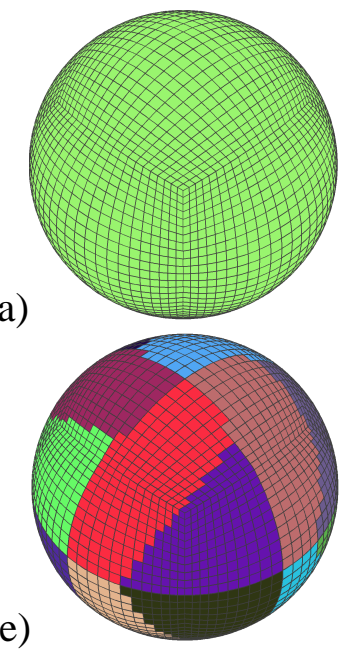

(b)
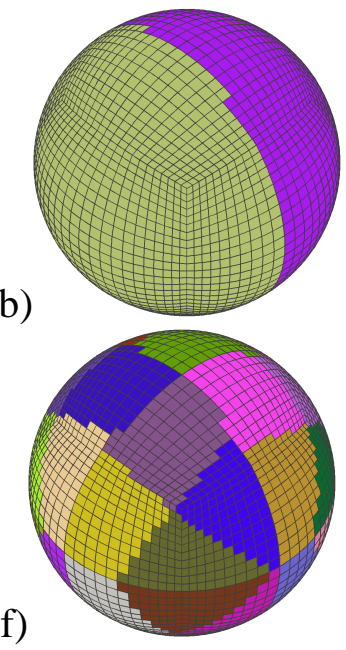

(c)
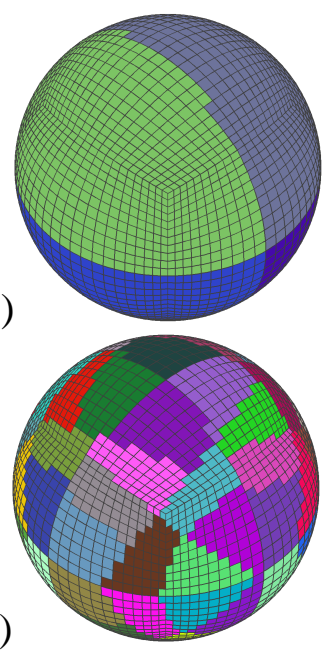

(d)

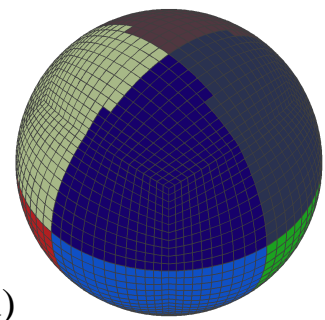

(h)

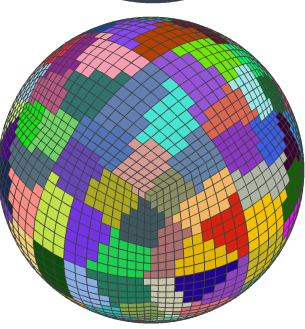

Figure 1. Clusters of (a) level 0, (b) level 1, (c) level 2, (d) level 3, (e) level 4, (f) level 5, (g) level 6 and (h) level 7.

The unit sphere is discretized by means of 3072 eight node quadrilateral boundary elements with element collocation (figure 1a). Both the classical and hierarchical BE method are used to calculate the response in a frequency range between $0 \mathrm{~Hz}$ and $100 \mathrm{~Hz}$, where analytical full space fundamental solutions [18] are employed. For the latter method, a hierarchical cluster tree is constructed based on the elements' center, as an element collocation scheme is used. A minimum number of elements $N_{\min }=24$ is specified, resulting in $\log _{2} \frac{3072}{24}=7$ cluster levels (figure 1). Figure 2 shows the hierarchical block structure of the matrices of $\hat{\mathbf{T}}_{\mathscr{H}}(\omega)$ and $\hat{\mathbf{U}}_{\mathscr{H}}(\omega)$ arising from this hierarchical clustering, where a value of 0.95 was attributed to the parameter $\eta$ in the admissibility criterion (2). A threshold $\varepsilon=10^{-3}$ has been used in the ACA algorithm to obtain low rank approximations of the blocks corresponding to admissible cluster pairs, while a tolerance of $10^{-4}$ was specified in the iterative GRMES solver.

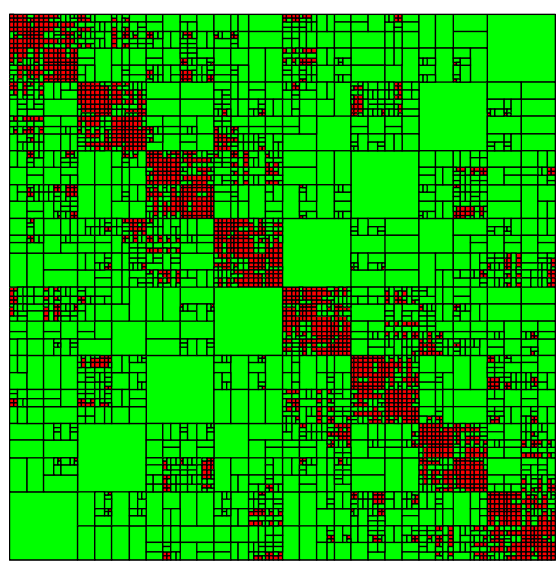

Figure 2. Hierarchical matrix decomposition. The green blocks correspond to admissible cluster pairs and are approximated by means of ACA, while the red blocks correspond to inadmissible cluster pairs and are computed exactly. 
Figure 3 shows the real and imaginary part of the radial displacement at the edge of the spherical cavity, calculated with the classical and the hierarchical BE method, respectively. A perfect agreement between the results of both methods can be observed. These results are furthermore compared to the analytical solution for the radial displacement in the full space, defined as [18]:

$$
\hat{u}_{r}(r, \omega)=\frac{r_{0}^{3}}{4 \rho C_{\mathrm{s}}^{2} r^{2}} \frac{1+i \omega_{\mathrm{p}}}{1+i \omega_{0}-\left(\omega_{0} \frac{C_{\mathrm{p}}}{2 C_{\mathrm{s}}}\right)^{2}} \exp \left(-i\left(\frac{r}{r_{0}}-1\right) \omega_{0}\right)
$$

where $\omega_{\mathrm{p}}=\omega r / C_{\mathrm{p}}$ and $\omega_{0}=\omega r_{0} / C_{\mathrm{p}}$. Equation (6) is evaluated for $r=1 \mathrm{~m}$. The results of both numerical methods correspond well with the analytical solution in the low frequency range, while some small deviations can be observed above $40 \mathrm{~Hz}$.

(a)

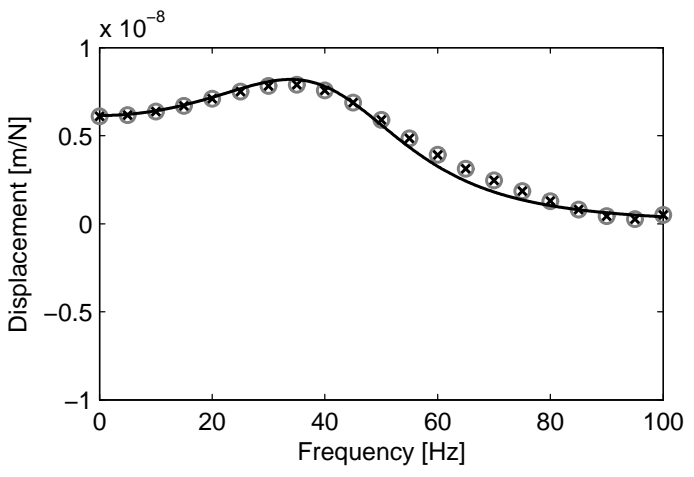

(b)

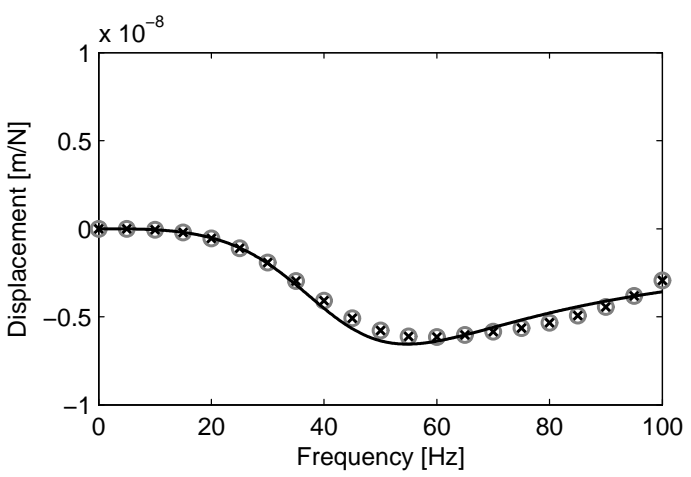

Figure 3. (a) Real and (b) imaginary part of the radial displacement at $\{r=1 \mathrm{~m}, \theta=0, z=$ $0 \mathrm{~m}\}$ due to a unit harmonic pressure applied at the edge of a spherical cavity with $r_{0}=1 \mathrm{~m}$. The solution of the hierarchical BE method (grey circles) is compared to the solution of the classical BE method (black crosses) and the analytical solution (solid line) [18].

The integral representation theorem subsequently allows to compute the radiated wavefield in the soil from the displacements and tractions on the boundary. Figure 4 shows the real and imaginary part of the radial displacement at the point $\{r=10 \mathrm{~m}, \theta=0, z=0 \mathrm{~m}\}$. It is clearly illustrated that the solutions of the classical and hierarchical BE method are in good correspondence, and agree with the analytical solution (i.e. equation (6) evaluated for $r=10 \mathrm{~m})$.

\subsection{Impedance of a massless rigid square surface foundation on a homogeneous half- space}

A massless rigid square surface foundation resting on a homogeneous halfspace is considered in this subsection. The foundation side equals $5 \mathrm{~m}$. The underlying halfspace has a shear wave velocity $C_{\mathrm{s}}=150 \mathrm{~m} / \mathrm{s}$, a dilatational wave velocity $C_{\mathrm{p}}=300 \mathrm{~m} / \mathrm{s}$, a density $\rho=1800 \mathrm{~kg} / \mathrm{m}^{3}$ and a material damping ratio $\beta_{\mathrm{s}}=\beta_{\mathrm{p}}=0.020$ in both deviatoric and volumetric deformation. The classical as well as the hierarchical BE method are employed to calculate the vertical soil impedance $\hat{K}_{z z}^{\mathrm{s}}(\omega)$, defined as:

$$
\hat{K}_{z z}^{\mathrm{s}}(\omega)=\int_{\Sigma} \boldsymbol{\psi}_{z z} \cdot \hat{\mathbf{t}}_{\mathrm{s}}^{\mathbf{n}_{\mathrm{s}}}\left(\hat{\mathbf{u}}_{\mathrm{sc}}\left(\boldsymbol{\psi}_{z z}\right) \mathrm{d} S\right.
$$



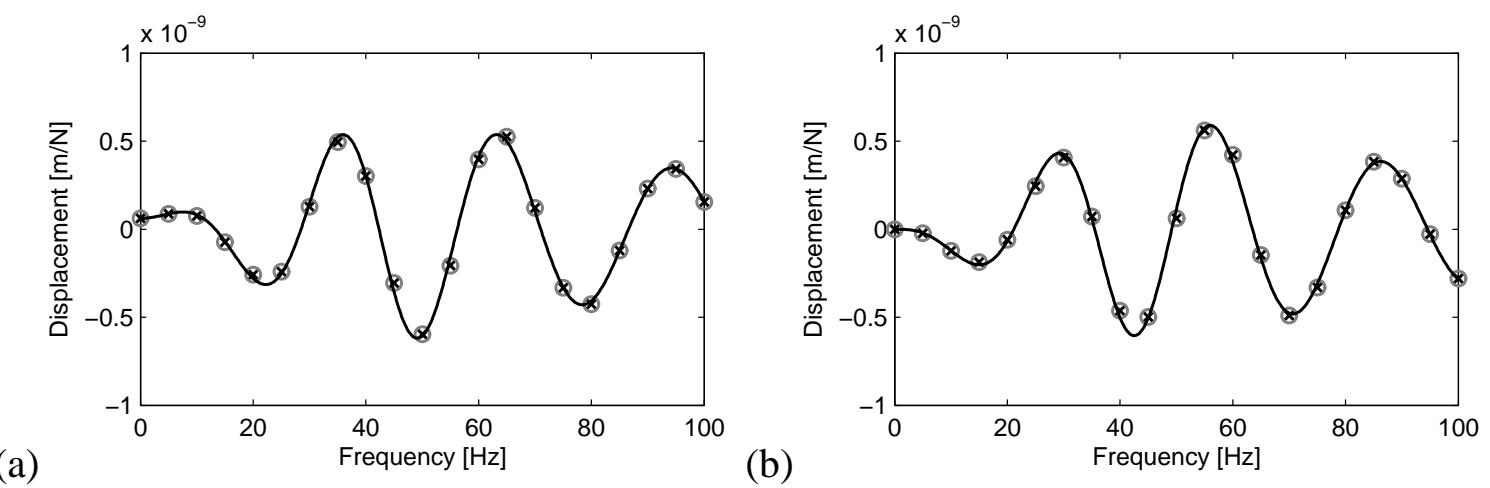

Figure 4. (a) Real and (b) imaginary part of the radial displacement at $\{r=10 \mathrm{~m}, \theta=0, z=$ $0 \mathrm{~m}\}$ due to a unit harmonic pressure applied at the edge of a spherical cavity with $r_{0}=1 \mathrm{~m}$. The solution of the hierarchical BE method (grey circles) is compared to the solution of the classical BE method (black crosses) and the analytical solution (solid line) [18].

where $\psi_{z z}$ indicates the vertical rigid body mode of the foundation. The soil-structure interface $\Sigma$ is discretized by means of four node quadrilateral boundary elements with element collocation. The tractions $\hat{\mathbf{t}}_{\mathrm{s}}^{\mathbf{n}_{\mathrm{s}}}\left(\hat{\mathbf{u}}_{\mathrm{sc}}\left(\boldsymbol{\psi}_{z z}\right)\right.$ due to imposed displacements $\boldsymbol{\psi}_{z z}$ are obtained by solving equations (1) and (5), respectively. As a surface foundation is considered, the system matrices $\hat{\mathbf{T}}(\omega)$ and $\hat{\mathbf{T}}_{\mathscr{H}}(\omega)$ vanish in these equations. The same values for the parameters $\eta, N_{\min }, \varepsilon$ and the tolerance in the GMRES solver as specified in subsection 3.1 are used in the hierarchical BE method. As mentioned in section 2, Green's functions for a halfspace are incorporated in both BE formulations [19, 25].

Figure 5a shows the real and imaginary part of the vertical soil impedance $\hat{K}_{z z}^{\mathrm{s}}(\omega)$ in a frequency range between $0 \mathrm{~Hz}$ and $100 \mathrm{~Hz}$. A BE mesh consisting of $30 \times 30$ equal sized elements is used to obtain these results. Up to nine elements per shear wavelength $\lambda_{\mathrm{s}}=C_{\mathrm{s}} / f$ are provided at the upper frequency of $100 \mathrm{~Hz}$. At high frequencies, the soil impedance $\hat{K}_{z z}^{\mathrm{s}}(\omega)$ converges to $i \omega \rho C_{\mathrm{s}} S$, where $S=5 \times 5 \mathrm{~m}^{2}$ is the area of the foundation [24]. A perfect match between the classical and hierarchical BE method can be observed.

(a)

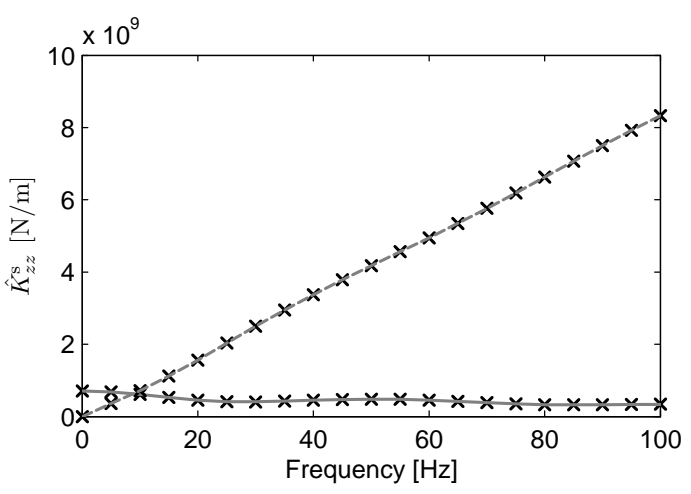

(b)

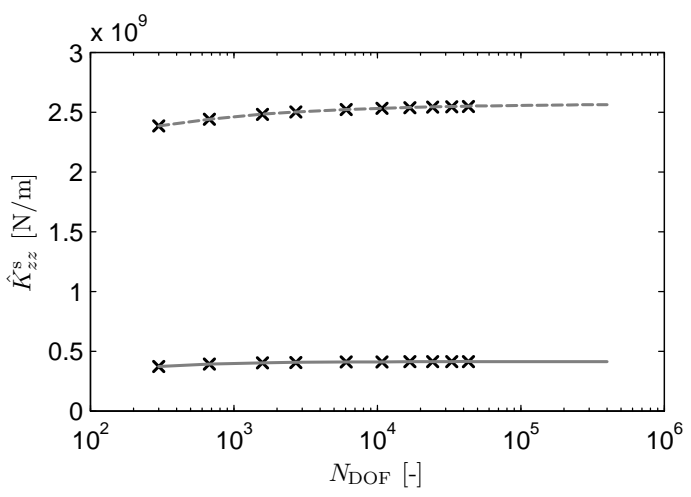

Figure 5. Real (solid line) and imaginary (dashed line) part of (a) the vertical soil impedance $\hat{K}_{z z}^{\mathrm{s}}(\omega)$ in function of frequency and (b) the vertical soil impedance $\hat{K}_{z z}^{\mathrm{s}}(\omega=2 \pi 30 \mathrm{~Hz})$ for an increasing number of degrees of freedom. The solution of the hierarchical BE method (grey lines) is compared to the solution of the classical BE method (black crosses). 
In order to demonstrate the effectiveness of the hierarchical BE method, the vertical soil impedance $\hat{K}_{z z}^{\mathrm{s}}(\omega)$ is reconsidered at a particular frequency of $30 \mathrm{~Hz}$, for an increasing number of boundary elements. As an element collocation scheme is applied, the number of degrees of freedom equals three times the number of elements. The real and imaginary part of $\hat{K}_{z z}^{\mathrm{s}}(\omega=2 \pi 30 \mathrm{~Hz})$ are shown in figure $5 \mathrm{~b}$. The results of both the hierarchical and classical BE method converge to a constant value $\hat{K}_{z z}^{\mathrm{s}}=(0.41+2.6 i) \times 10^{9} \mathrm{~N} / \mathrm{m}$ due to the mesh refinement, and are in good correspondence with each other. The application of the classical BE method is, however, limited to a model size of 43200 degrees of freedom. The storage of the complex floating point entries of $\hat{\mathbf{U}}(\omega)$ in double precision requires in that case $2 \times N_{\text {DOF }}^{2} \times 8$ bytes $=27.8$ GB of RAM memory, being the limit of the machine on which the actual calculations have been performed. Figure 6a compares the memory required to store the BE matrices $\hat{\mathbf{U}}(\omega)$ and $\hat{\mathbf{U}}_{\mathscr{H}}(\omega)$ on a double logarithmic plot, in which the slope of the curve indicates the power of the number of degrees of freedom to which the memory is proportional to. As expected, a quadratic trend $\mathcal{O}\left(N_{\mathrm{DOF}}^{2}\right)$ is retrieved for the classical BE method. For the hierarchical BE method, however, the memory requirement is of the order $\mathcal{O}\left(N_{\text {DOF }} \log _{10}^{3} N_{\text {DOF }}\right)$, allowing to extend the model size up to 399675 degrees of freedom with only $28 \mathrm{~GB}$ of RAM memory available. In comparison, $2380 \mathrm{~GB}$ of RAM would be required in order to handle such a model with the classical BE method. Figure $6 \mathrm{~b}$ shows the CPU time required to calculate $\hat{K}_{z z}^{\mathrm{s}}(\omega=2 \pi 30 \mathrm{~Hz})$, including both the time to assemble to matrices $\hat{\mathbf{U}}(\omega)$ or $\hat{\mathbf{U}} \mathscr{H}(\omega)$ and to solve the set of equations (1) or (5), respectively. For relatively small models $\left(\mathcal{O}\left(10^{3}\right)\right)$, the classical BE method turns out to be a little faster, while from a moderate model size on, the hierarchical approach becomes faster.
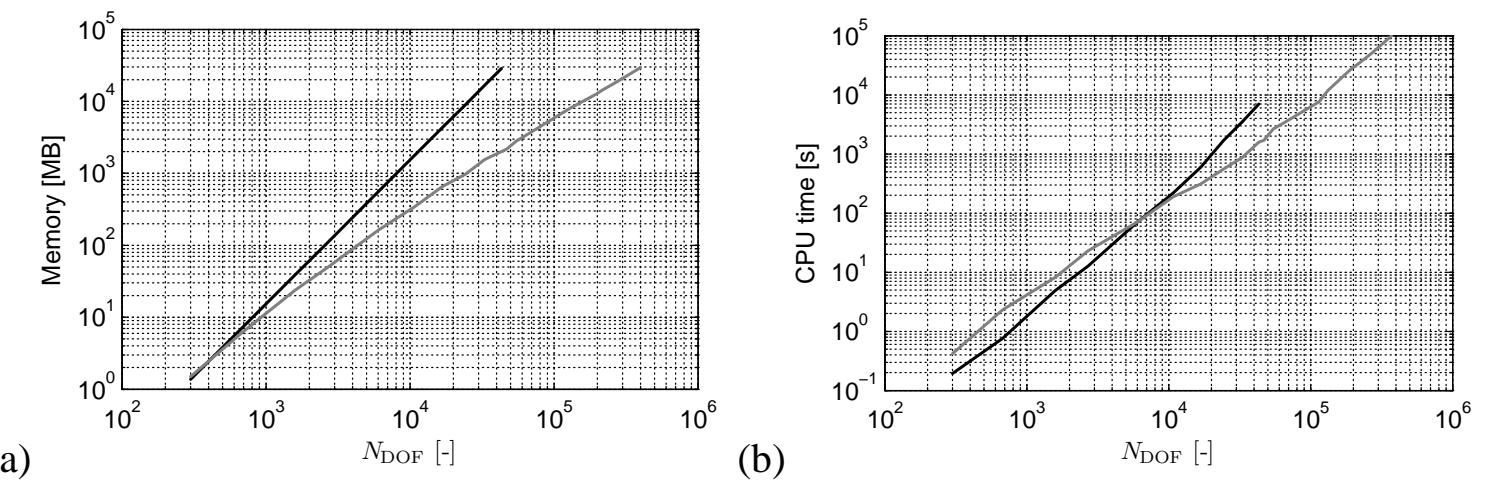

Figure 6. (a) RAM memory and (b) total CPU time required for an increasing number of degrees of freedom with the classical (black lines) and the hierarchical (grey lines) BE method.

The contribution of the assembly time and the solution time to the total CPU time is further investigated in figure 7. For the classical BE method, the assembly time shows a quadratic trend. The solution time of the direct solver, however, increases in a cubic way. The time required to solve the set of equations (1) will therefore dominate the total solution time of the classical BE method for large models. Figure 7 also illustrates, on the other hand, that the contribution of the solution time is negligible compared to the assembly time for the hierarchical BE method. No effort has therefore been made to incorporate a preconditioner in the iterative GMRES solver, such as an $\mathscr{H}-L U$ preconditioner proposed in [5], as speeding up of the iterative solver will not result in a significant reduction of the total CPU time required in the hierarchical BE method. 


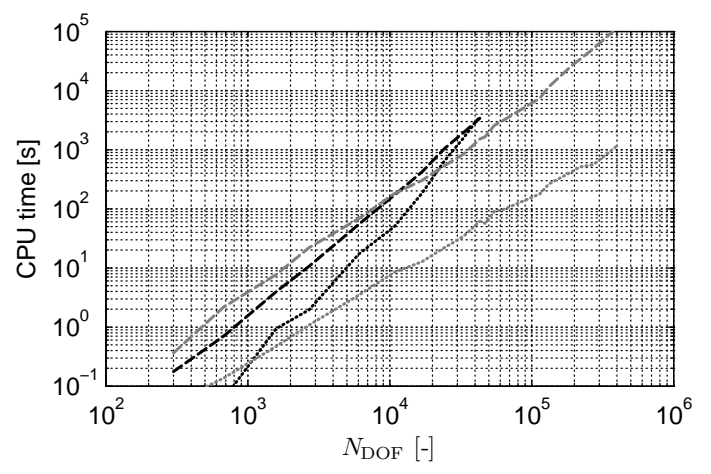

Figure 7. CPU time required to assemble the matrices $\hat{\mathbf{U}}(\omega)$ or $\hat{\mathbf{U}}_{\mathscr{H}}(\omega)$ (dashed lines) and to solve the set of equations (1) or (5) (dotted lines) with the classical (black) and the hierarchical (grey) BE method.

The example discussed in this subsection illustrates that the hierarchical BE method combined with Green's functions for a horizontally layered halfspace is very efficient, very fast and sufficiently accurate. The proposed approach is advantageous with respect to existing fast BE methods for elastodynamics involving a layered halfspace, such as the FMM [9, 15], as these formulations are based on full space Green's functions, and hence need to sacrifice a considerable amount of boundary elements to the modelling of the free surface and the layer interfaces. This consequently poses a restriction on the size of the actual problem which can be treated. Furthermore, the hierarchical BE method does not need any modification to tackle visco-elastodynamic problems (as illustrated in this example), while a damping dependent modification of the selection rule for the multipole truncation parameter is required in the FMM [15].

\section{CASE STUDY}

The examples put forward in the previous section validate the proposed hierarchical BE method, and this eventually allows to predict railway induced vibrations in an urban environment. In this section, a synthetic case is therefore introduced to investigate the influence of the trough-soil coupling of closely spaced buildings.

\subsection{Model description}

The synthetic case study consists of a set of 12 identical masonry buildings resting on a homogeneous halfspace (figure 8a). Each masonry building has dimensions $12 \mathrm{~m} \times$ $6 \mathrm{~m} \times 8 \mathrm{~m}$ and has stories, each subdivided into 4 rooms. The interior and exterior walls have a thickness $t_{\mathrm{w}}=0.10 \mathrm{~m}$, and consist of clay brick masonry. The floors are concrete slabs with a thickness $t_{\mathrm{fl}}=0.20 \mathrm{~m}$. All floors are simply supported, corresponding to hinged joints at the slab edges. The structure is founded on a concrete strip foundation with a width $w_{\mathrm{f}}=0.60 \mathrm{~m}$ and a thickness $t_{\mathrm{f}}=0.20 \mathrm{~m}$ [10]. The buildings are modelled with the finite element method. The strip foundation, the walls and the floors are modelled by means of shell elements, using isotropic properties for the foundation and the floors and orthotropic properties for the masonry walls. The lintels above the door and the windows are modelled by means of beam elements [10]. 

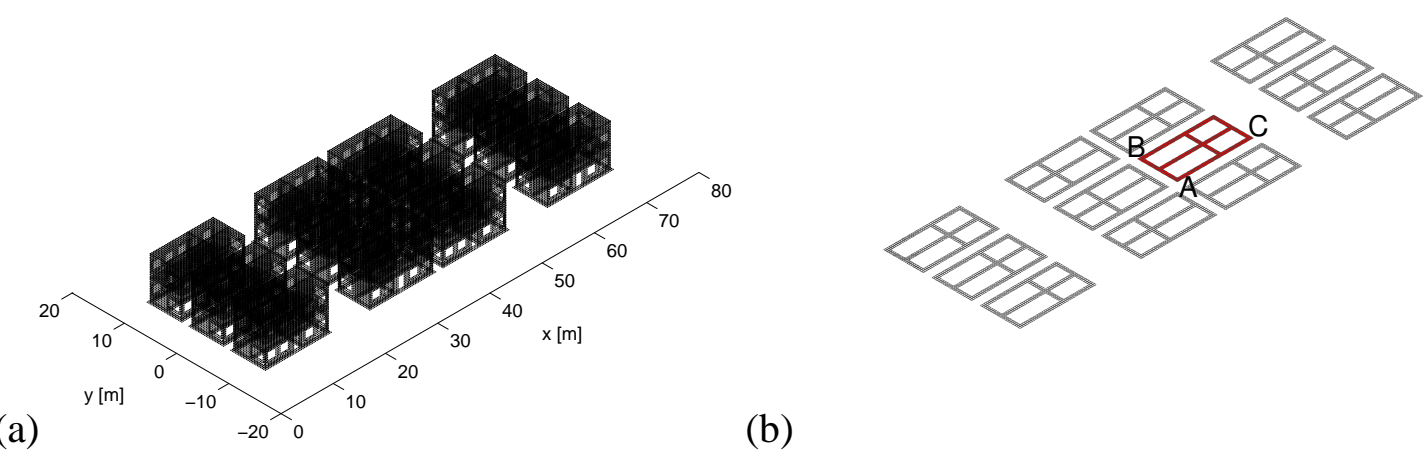

(b)

Figure 8. (a) Finite element and (b) boundary element mesh of the set of 12 masonry buildings.

The front edge of the first set of three buildings is located at $x=6 \mathrm{~m}$, with a separation distance of $2 \mathrm{~m}$ in the $y$-direction between the buildings. A gap of $8 \mathrm{~m}$ in the $x$-direction exists between this set and the following set of three buildings. Six additional buildings are furthermore included behind this second set, in a same pattern as the previous six buildings.

The homogeneous halfspace is characterized by a shear wave velocity $C_{\mathrm{s}}=200 \mathrm{~m} / \mathrm{s}$, a dilatational wave velocity $C_{\mathrm{p}}=400 \mathrm{~m} / \mathrm{s}$, a density $\rho=1800 \mathrm{~kg} / \mathrm{m}^{3}$ and a material damping ratio $\beta_{\mathrm{s}}=\beta_{\mathrm{p}}=0.025$ in both deviatoric and volumetric deformation. An incident wavefield is generated by a unit vertical point load acting on the surface of the halfspace at the origin of the coordinate system.

\subsection{Coupled FE-BE model}

A coupled FE-BE methodology formulated in the frequency domain which accounts for dynamic SSI is employed to calculate the response of the buildings to the incident wavefield. If $\mathrm{N}$ structures are considered, a weak variational formulation of the equilibrium of structure $j(j=1, \ldots, \mathrm{N})$ and a subdomain formulation [2,3] results in the following set of coupled FE-BE equations:

$$
\left[\mathbf{K}_{j}+i \omega \mathbf{C}_{j}-\omega^{2} \mathbf{M}_{j}\right] \underline{\hat{\mathbf{u}}}_{j}(\omega)+\sum_{k=1}^{\mathrm{N}} \hat{\mathbf{K}}_{j k}^{\mathrm{s}}(\omega) \underline{\hat{\mathbf{u}}}_{k}(\omega)=\underline{\hat{\mathbf{f}}}_{j}^{\mathrm{s}}(\omega) \quad \text { for } \quad j=1 \ldots \mathrm{N}
$$

$\underline{\hat{\mathbf{u}}}_{j}(\omega)$ collects the nodal degrees of freedom of structure $j$, while $\mathbf{K}_{j}, \mathbf{C}_{j}$ and $\mathbf{M}_{j}$ are the stiffness, damping and mass matrix of this structure. Rayleigh damping is assumed for the damping matrix $\mathbf{C}_{j}[10] . \hat{\mathbf{K}}_{j k}^{\mathrm{s}}(\omega)$ are the dynamic stiffness matrices of the soil, which represent the through-soil coupling of structures $j$ and $k$ for $j \neq k$. The force vector $\underline{\hat{\mathbf{f}}}_{j}^{\mathrm{s}}(\omega)$ denotes the dynamic soil-structure interaction forces at the soil-structure interface $\Sigma_{j}$ due to the incident wave field. A Craig-Bampton substructuring technique is furthermore used, decomposing each structure $j$ into the foundation and the superstructure, and an appropriate modal decomposition is introduced [10].

The hierarchical BE method outlined in section 2 is employed to evaluate the dynamic soil stiffness matrices $\hat{\mathbf{K}}_{j k}^{\mathrm{s}}(\omega)$ and the force vectors $\underline{\hat{\mathbf{f}}}_{j}^{\mathrm{s}}(\omega)$ arising from the incident wave field. The finite elements of the foundations are coupled to a conforming boundary element mesh for the surrounding soil (figure 8b), and a nodal collocation scheme is used to facilitate the FE$\mathrm{BE}$ coupling. As a nodal collection scheme is used, the hierarchical clustering is based on the 
nodes rather than on the elements' center. The same values for the parameters $\eta, N_{\min }, \varepsilon$ and the tolerance in the GMRES solver as specified in subsection 3.1 are used in the hierarchical BE method (where $N_{\min }$ indicates in this case a minimum number of nodes).

It must also be stressed that a full 3D calculation is performed, without introducing any additional assumptions concerning the lay-out of the buildings (i.e. no periodicity considerations are taken into account).

\subsection{Results}

First, the response of the set of 12 buildings to an incident wavefield generated by a unit vertical harmonic point load at $10 \mathrm{~Hz}$ is considered. At this frequency, the Rayleigh wavelength in the soil equals $\lambda_{\mathrm{R}}=C_{\mathrm{R}} / f=18.6 \mathrm{~m}$. The incident wave field, characterized by cylindrical wave fronts, is shown in figure $9 \mathrm{a}$. Figure $9 \mathrm{~b}$ shows the wave field in the soil in case the presence of all 12 buildings is simultaneously taken into account. As the Rayleigh wavelength is larger than the dimensions of the strip foundations, the wave field remains nearly cylindrically.

(a)

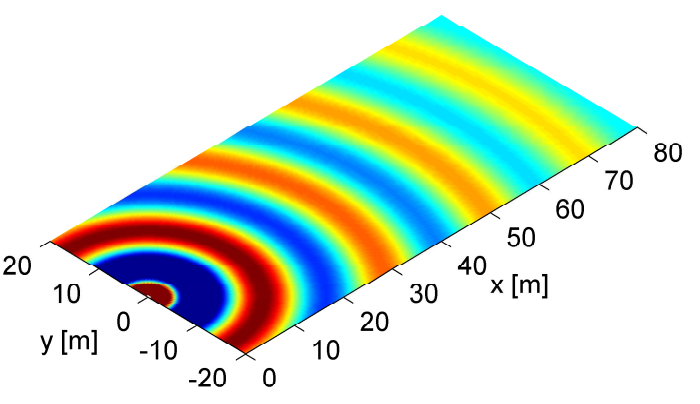

(b)

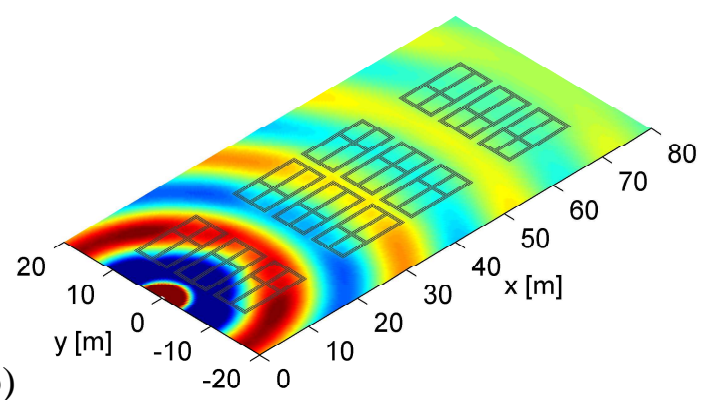

Figure 9. Real part of the vertical soil displacement field $\hat{u}_{z}$ at $10 \mathrm{~Hz}$ (a) without and (b) with accounting for the presence of the buildings.

The influence of the through-soil coupling on the structural response is illustrated in figure 10 .

(a)

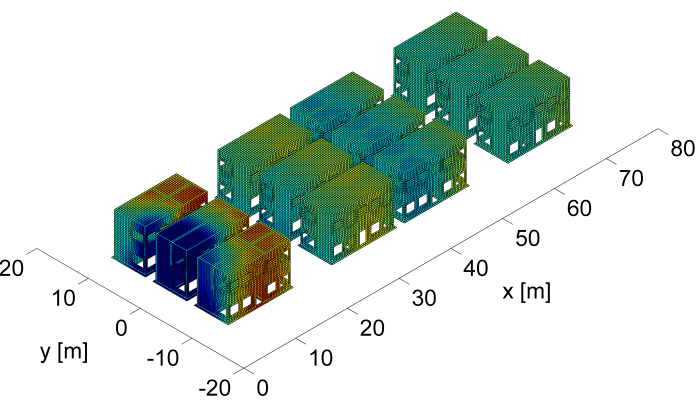

20

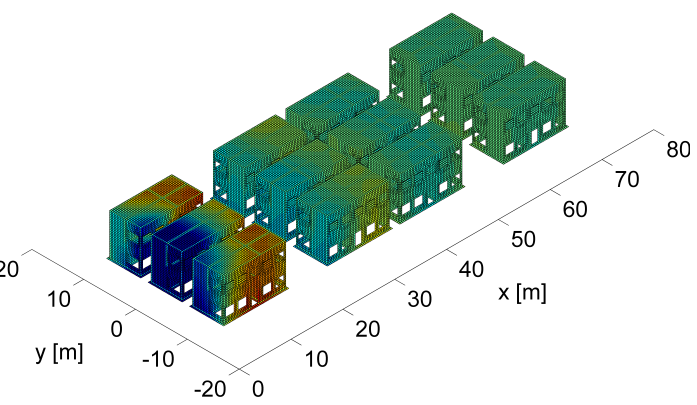

Figure 10. Real part of the vertical structural displacement field $\hat{u}_{z}$ at $10 \mathrm{~Hz}$ (a) without and (b) with accounting for the through-soil coupling of the surrounding buildings.

Figure 10a shows the vertical response of all buildings, in case the presence of the surrounding buildings is neglected for each building. This is obtained by solving the set of equations (8) $N$ times, in which the soil stiffness matrices $\hat{\mathbf{K}}_{j k}^{\mathrm{s}}(\omega)$ are not considered for $j \neq k$. Figure $10 \mathrm{~b}$ illustrates the structural response in case the presence of all 12 buildings is simultaneously 
taken into account. A visual comparison of figures $10 \mathrm{a}$ and $10 \mathrm{~b}$ indicates that the structural response is qualitatively similar, especially for the three buildings closest to the source of the incident wavefield. The response of the buildings further away from the source turns out to be more affected by the presence of the surrounding buildings.

The influence of the through-soil coupling on the structural response is investigated in more detail for one particular building, which is indicated in red on figure 8b. Figure 11 compares the modulus and the phase of the vertical displacement $\hat{u}_{z}$ along the front wallfoundation edge $\mathrm{AB}$. The response is not symmetrical due to the non-symmetrical layout of the individual masonry buildings (i.e. the presence of the door and windows). While the variation of the displacements along the edge is similar in both cases, the amplitude is almost halved if the through-soil coupling is accounted for. This indicates that the buildings closer to the source shield the considered building from the incident wavefield. Furthermore, an almost constant phase shift of $1.35 \mathrm{rad}$ is introduced along the edge $\mathrm{AB}$, as the Rayleigh wave fronts are slightly shifted due the the presence of the buildings. Similarly, figure 12 compares the modulus and the phase of the vertical displacement $\hat{u}_{z}$ along the side wall-foundation edge AC. The variation of the displacements along the edge is similar in both cases; an amplitude reduction by a factor of 2 and a phase shift of $1.35 \mathrm{rad}$ can be observed here as well.

(a)

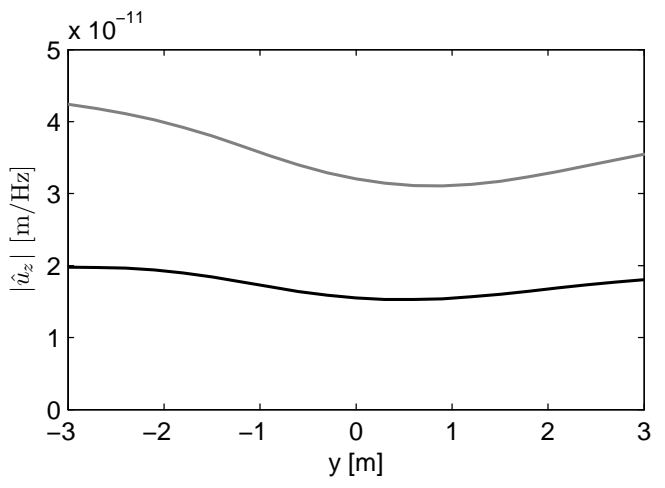

(b)

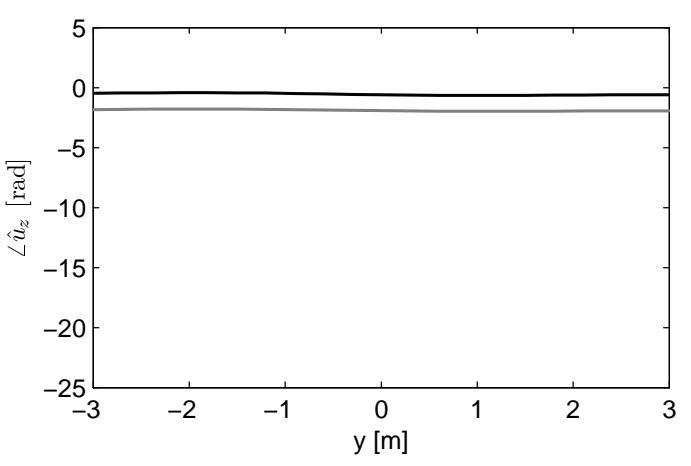

Figure 11. (a) Modulus and (b) phase of the vertical displacement $\hat{u}_{z}$ along the front wallfoundation edge $\mathrm{AB}$ at $10 \mathrm{~Hz}$ with (black lines) and without (grey lines) accounting for the through-soil coupling of the surrounding buildings.

(a)

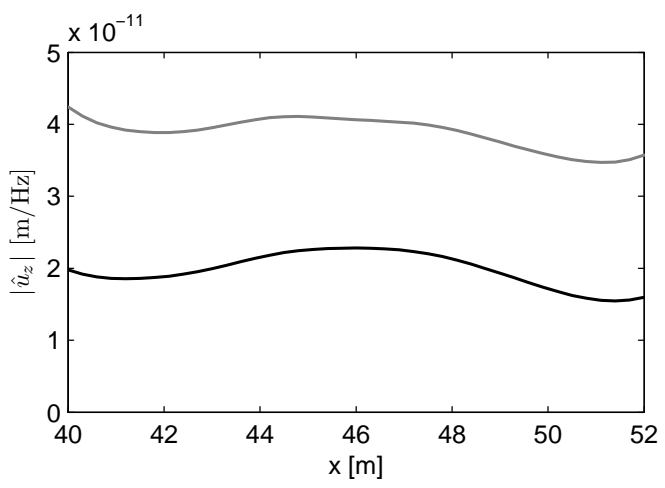

(b)

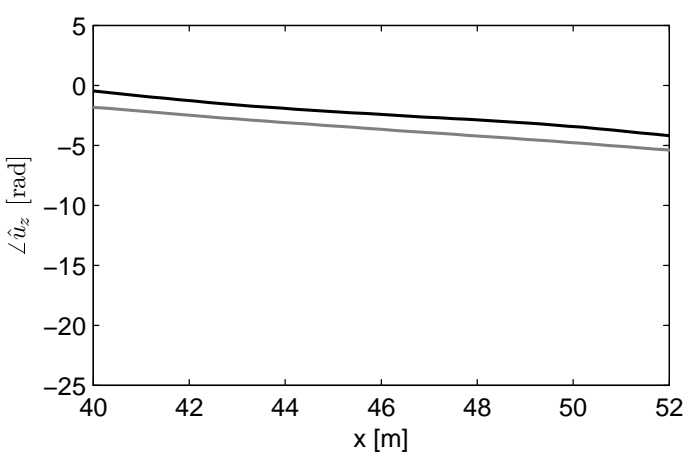

Figure 12. (a) Modulus and (b) phase of the vertical displacement $\hat{u}_{z}$ along the side wallfoundation edge $\mathrm{AC}$ at $10 \mathrm{~Hz}$ with (black lines) and without (grey lines) accounting for the through-soil coupling of the surrounding buildings. 
Second, the response of the set of 12 buildings to an incident wavefield generated by a unit vertical harmonic point load at $50 \mathrm{~Hz}$ is considered. At $50 \mathrm{~Hz}$, the Rayleigh wavelength in the soil equals $\lambda_{\mathrm{R}}=C_{\mathrm{R}} / f=3.7 \mathrm{~m}$. The incident wave field, characterized by cylindrical wave fronts, is shown in figure 13a. The dynamic interaction between the buildings and the halfspace alters the wave field significantly (figure 13b), as the wavelength in the soil becomes of the same order of magnitude as the dimensions of the strip foundations.

(a)

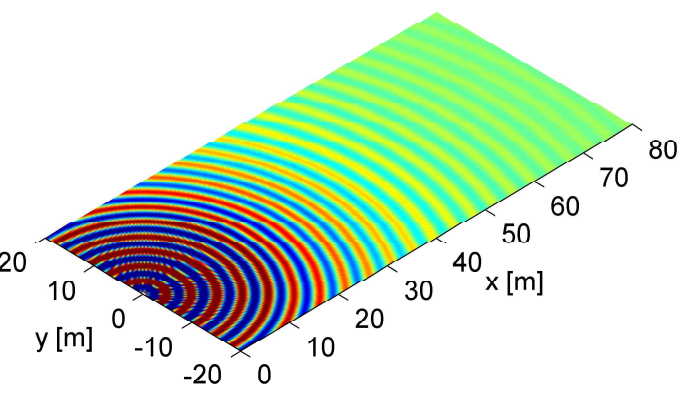

(b)

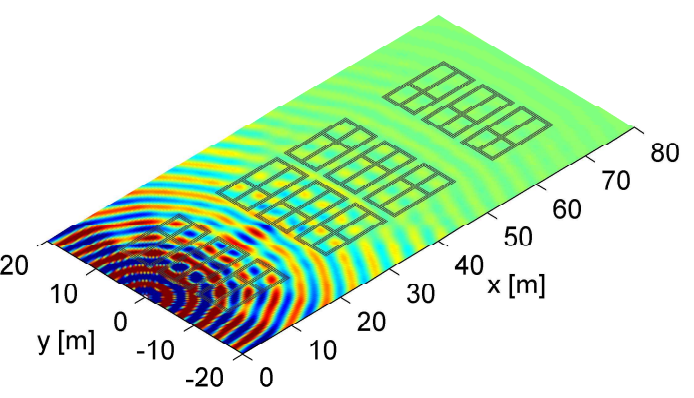

Figure 13. Real part of the vertical soil displacement field $\hat{u}_{z}$ at $50 \mathrm{~Hz}$ (a) without and (b) with accounting for the presence of the buildings.

The influence of the through-soil coupling on the structural response is illustrated in figure 14 .

(a)

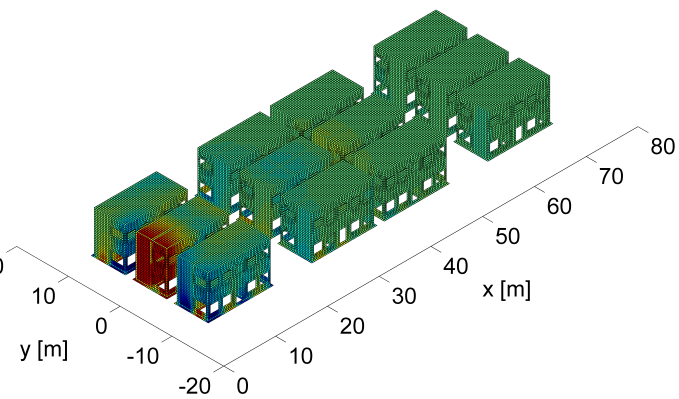

20

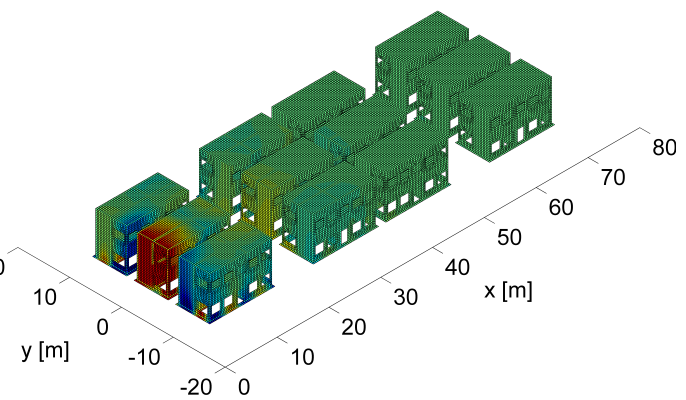

Figure 14. Real part of the vertical structural displacement field $\hat{u}_{z}$ at $50 \mathrm{~Hz}$ (a) without and (b) with accounting for the through-soil coupling of the surrounding buildings.

Figure 14a shows the vertical response of all buildings, in case the presence of the surrounding buildings is neglected for each building, while figure $14 \mathrm{~b}$ illustrates the structural response in case the presence of all 12 buildings is simultaneously taken into account. As in figure 9, the response of the three buildings closest to the source of the incident wavefield remains almost unaffected. A visual comparison of the other buildings, however, suggests that the response is considerably altered by the presence of the surrounding buildings.

The influence of the through-soil coupling on the structural response is investigated in more detail for the same particular building as discussed before. Figure 15 compares the modulus and the phase of the vertical displacement $\hat{u}_{z}$ along the front wall-foundation edge $\mathrm{AB}$. The amplitude is reduced by more than a factor of 2 , and an an almost constant phase shift of $3.3 \mathrm{rad}$ is induced. Figure 16 compares the modulus and the phase of the vertical displacement $\hat{u}_{z}$ along the side wall-foundation edge AC. Not only can an amplitude reduction with respect to the case where the through-soil coupling of the surrounding buildings is 
neglected be observed, but the variation of vertical displacements along this edge also differs significantly. A phase shift of $3.3 \mathrm{rad}$ is introduced as well.

(a)

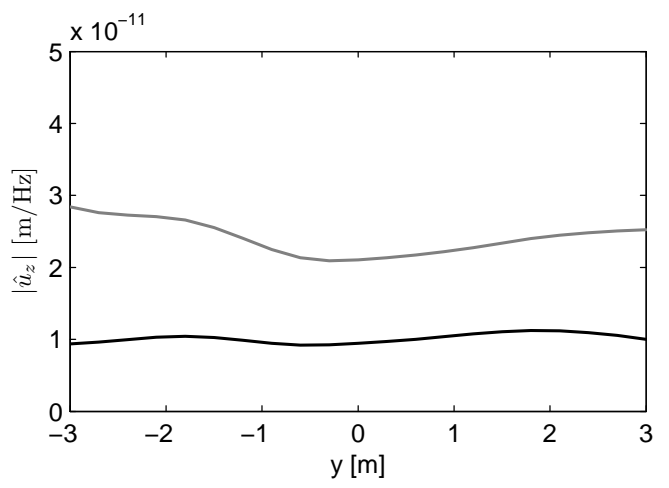

(b)

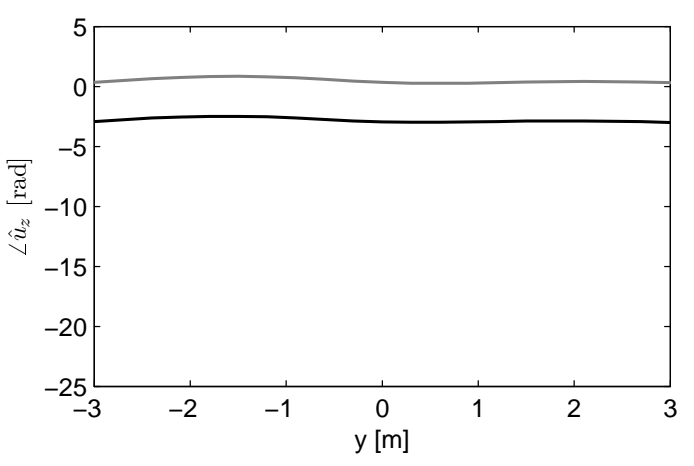

Figure 15. (a) Modulus and (b) phase of the vertical displacement $\hat{u}_{z}$ along the front wallfoundation edge $\mathrm{AB}$ at $50 \mathrm{~Hz}$ with (black lines) and without (grey lines) accounting for the through-soil coupling of the surrounding buildings.

(a)

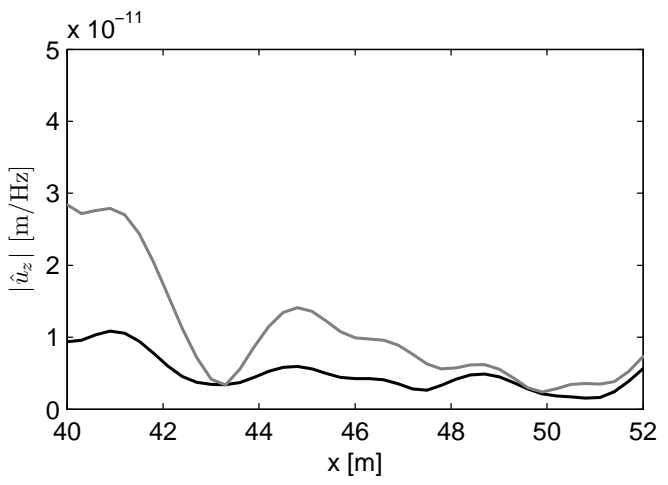

(b)

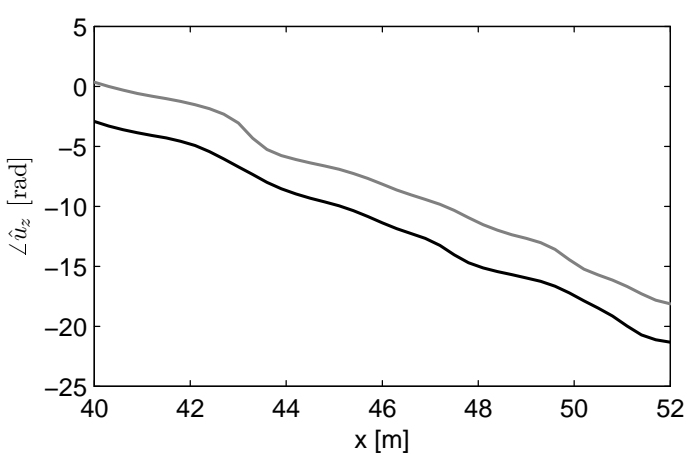

Figure 16. (a) Modulus and (b) phase of the vertical displacement $\hat{u}_{z}$ along the side wallfoundation edge $\mathrm{AC}$ at $50 \mathrm{~Hz}$ with (black lines) and without (grey lines) accounting for the through-soil coupling of the surrounding buildings.

Although only a limited number of results have been discussed in this subsection, the presented synthetic case study nevertheless indicates that the wave propagation in the soil and the structural response is considerably affected in an urban environment. At low frequencies, this predominantly results in a shielding effect, reducing the amplitudes of the displacements, without altering the wavefield itself drastically. At higher frequencies, however, the wavelength in the soil becomes comparable to the foundation dimensions, and the dynamic interaction between the buildings and the halfspace alters the wavefield significantly.

\section{CONCLUSIONS}

In this paper, a hierarchical BE method based on Green's functions for a horizontally layered halfspace is presented. The numerical implementation has been validated, and it has been demonstrated that the proposed methodology is very efficient, very fast and sufficiently 
accurate, which allows to perform large scale BE computations involving a layered halfspace. The applicability of the novel methodology has been illustrated by means of a synthetic case study of an urban environment, indicating that the through-soil coupling of closely spaced buildings can significantly alter the wave propagation in the soil and the structural response.

\section{Acknowledgements}

The first author is a doctoral fellow and the second author is a postdoctoral fellow of the Research Foundation Flanders (FWO). The financial support is gratefully acknowledged.

\section{References}

[1] L. Andersen and C.J.C. Jones. Coupled boundary and finite element analysis of vibration from railway tunnels - a comparison of two- and three-dimensional models. Journal of Sound and Vibration, 293(3-5):611-625, 2006.

[2] D. Aubry and D. Clouteau. A subdomain approach to dynamic soil-structure interaction. In V. Davidovici and R.W. Clough, editors, Recent advances in Earthquake Engineering and Structural Dynamics, pages 251-272. Ouest Editions/AFPS, Nantes, 1992.

[3] D. Aubry, D. Clouteau, and G. Bonnet. Modelling of wave propagation due to fixed or mobile dynamic sources. In N. Chouw and G. Schmid, editors, Workshop Wave '94, Wave propagation and Reduction of Vibrations, pages 109-121, Ruhr Universität Bochum, Germany, December 1994.

[4] M. Bebendorf. Approximation of boundary element matrices. Numerische Mathematik, $86: 565-589,2000$.

[5] M. Bebendorf. Hierarchical $L U$ decomposition based preconditioners for BEM. Computing, 74:225-247, 2005.

[6] M. Bebendorf. Hierarchical Matrices: A Means to Efficiently Solve Elliptic Boundary Value Problems. Springer Publishing Company, 1st edition, 2008.

[7] M. Bebendorf and S. Rjasanow. Adaptive low-rank approximation of collocation matrices. Computing, 70:1-24, 2003.

[8] I. Benedetti and M. H. Aliabadi. A fast hierarchical dual boundary element method for three-dimensional elastodynamic crack problems. International Journal for Numerical Methods in Engineering, 84(9):1038-1067, 2010.

[9] S. Chaillat, M. Bonnet, and J. Semblat. A new fast multi-domain BEM to model seismic wave propagation and amplification in 3D geological structures. Geophysical Journal International, 177:509-531, 2009.

[10] S. François. Nonlinear modelling of the response of structures due to ground vibrations. $\mathrm{PhD}$ thesis, Department of Civil Engineering, K.U.Leuven, 2008.

[11] S. François, M. Schevenels, and G. Degrande. BEMFUN: MATLAB toolbox for boundary elements in elastodynamics. Version 2.1 Build 16. User's guide BWM-2009-26, Department of Civil Engineering, K.U.Leuven, December 2009. 
[12] A. George. Nested dissection of a regular finite element mesh. SIAM Journal on Numerical Analysis, 10:345-363, 1973.

[13] L. Grasedyck and W. Hackbusch. Construction and arithmetics of $\mathscr{H}$-matrices. Computing, 70:295-334, 2003.

[14] L. Grasedyck, R. Kriemann, and S. Le Borne. Domain decomposition based $\mathscr{H}-L U$ preconditioning. Numerische Mathematik, 112(4):565-600, 2009.

[15] E. Grasso, S. Chaillat, M. Bonnet, and J. Semblat. Application of the multi-level timeharmonic fast multipole BEM to 3-D visco-elastodynamics. Engineering Analysis with Boundary Elements, 36(5):744-758, 2012.

[16] W. Hackbusch. A sparse matrix arithmetic based on $\mathscr{H}$-matrices. Part I: Introduction to $\mathscr{H}$-matrices. Computing, 62(2):89-108, 1999.

[17] W. Hackbusch and Z.P. Nowak. On the fast matrix multiplication in the boundary element method by panel clustering. Numerische Mathematik, 54:463-491, 1989.

[18] E. Kausel. Fundamental solutions in elastodynamics: a compendium. Cambridge University Press, New York, 2006.

[19] E. Kausel and J.M. Roësset. Stiffness matrices for layered soils. Bulletin of the Seismological Society of America, 71(6):1743-1761, 1981.

[20] G. Lombaert, G. Degrande, J. Kogut, and S. François. The experimental validation of a numerical model for the prediction of railway induced vibrations. Journal of Sound and Vibration, 297(3-5):512-535, 2006.

[21] A. Milazzo, I. Benedetti, and M.H. Aliabadi. Hierarchical fast BEM for anisotropic time-harmonic 3-D elastodynamics. Computers and Structures, 96-97:9-24, 2012.

[22] S. Rjasanow and O. Steinbach. The Fast Solution of Boundary Integral Equations (Mathematical and Analytical Techniques with Applications to Engineering). Springer-Verlag New York, 2007.

[23] V. Rokhlin. Rapid solution of integral equations of classical potential theory. Journal of Computational Physics, 60:187-207, 1985.

[24] M. Schevenels. The impact of uncertain dynamic soil characteristics on the prediction of ground vibrations. PhD thesis, Department of Civil Engineering, K.U.Leuven, 2007.

[25] M. Schevenels, S. François, and G. Degrande. EDT: An ElastoDynamics Toolbox for MATLAB. Computers \& Geosciences, 35(8):1752-1754, 2009. 